1997-01-01

\title{
Tradeoffs in the design of a spaceborne scanning pencil beam scatterometer: Application to SeaWinds
}

David G. Long

david_long@byu.edu

Chialin T. Wu

Michael W. Spencer

Follow this and additional works at: https://scholarsarchive.byu.edu/facpub

Part of the Electrical and Computer Engineering Commons

\section{Original Publication Citation}

Spencer, M. W., Chialin Wu, and D. G. Long. "Tradeoffs in the Design of a Spaceborne Scanning Pencil Beam Scatterometer: Application to SeaWinds." Geoscience and Remote Sensing, IEEE Transactions on 35.1 (1997): 115-26

\section{BYU ScholarsArchive Citation}

Long, David G.; Wu, Chialin T.; and Spencer, Michael W., "Tradeoffs in the design of a spaceborne scanning pencil beam scatterometer: Application to SeaWinds" (1997). Faculty Publications. 674.

https://scholarsarchive.byu.edu/facpub/674

This Peer-Reviewed Article is brought to you for free and open access by BYU ScholarsArchive. It has been accepted for inclusion in Faculty Publications by an authorized administrator of BYU ScholarsArchive. For more information, please contact ellen_amatangelo@byu.edu. 


\title{
Tradeoffs in the Design of a Spaceborne Scanning Pencil Beam Scatterometer: Application to SeaWinds
}

\author{
Michael W. Spencer, Chialin Wu, and David G. Long
}

\begin{abstract}
SeaWinds is a spaceborne wind scatterometer to be flown on the second Japanese Advanced Earth Observing Satellite (ADEOS-II) in 1999. An important international element of NASA's earth observing system (EOS), SeaWinds is an advanced follow-on to the NASA scatterometer (NSCAT) on the first ADEOS platform. Unlike previous operational spaceborne scatterometer systems, SeaWinds employs a scanning "pencilbeam" antenna rather than a "fan-beam" antenna, making the instrument more compact and yielding greater ocean coverage. The goals of this paper are twofold. First, the overall SeaWinds functional design and backscatter measurement approach are described, and the relative advantages of the pencil-beam technique are outlined. Second, the unique aspects of measurement accuracy optimization and signal processing for the SeaWinds instrument are discussed. Applying the results of a separate companion paper [11], a technique to significantly improve measurement accuracy by modulating the transmit pulse is described. Trade-offs to optimize the transmit modulation bandwidth are presented.
\end{abstract}

Index Terms - Radar, scatterometry, SeaWinds, wind measurement.

\section{INTRODUCTION}

$\mathbf{T}$ HE SeaWinds scatterometer will be launched aboard the second Japanese Advanced Earth Observing Satellite (ADEOS-II) in early 1999. SeaWinds, as part of the NASA earth observing system (EOS), will acquire all-weather measurements of ocean wind speed and direction. SeaWinds observations will continue the $\mathrm{Ku}$-Band scatterometer data base begun by NSCAT [14] into the next century. Because knowledge of near-surface winds over the ocean is critical for the investigation of many oceanographic and meteorological phenomena [3], SeaWinds observations form a key element of the EOS climatological research mission. In addition to its scientific applications, SeaWinds is expected to provide an important data source for operational meteorologists. Accurate and timely observations of ocean winds will enhance the ability of forecasters to identify coastal and marine hazards.

As with all scatterometers, SeaWinds will obtain an estimate of the wind by measuring the ocean surface radar backscatter cross section $\left(\sigma^{0}\right)$ at multiple azimuth angles. The geophysical model function, which relates wind speed and direction to backscatter cross section, is then numerically inverted to infer the near surface wind. This technique has been successfully employed on previous scatterometer programs,

Manuscript received September 21, 1995; revised May 15, 1996.

M. W. Spencer and C. Wu are with the Jet Propulsion Laboratory, California Institute of Technology, Pasadena CA 91109 USA.

D. G. Long is with the Electrical and Computer Engineering Department, Brigham Young University, Provo UT 84602 USA.

Publisher Item Identifier S 0196-2892(97)00969-8. such as SEASAT and ERS-1, and is planned for SeaWinds' direct predecessor, NSCAT [1], [5], [14]. Unlike these earlier scatterometers which employed a fixed "fan-beam" antenna, however, SeaWinds will utilize a scanning "pencil-beam" design.

In fan-beam systems, sticklike antennas are used to broadcast long, narrow radar footprints on the ocean surface. Along track $\sigma^{0}$ resolution is defined by the narrow dimension of the footprint, and cross track resolution is obtained by either Doppler or range discrimination. Typically, a 500-600 km swath of measurements can be obtained on either side of the spacecraft, but in the region $\pm 200 \mathrm{~km}$ from the satellite nadir track, wind vectors cannot be measured due to the inappropriately low incidence angles. The existence of this "nadir gap" significantly limits ocean coverage. Fan-beam systems are also difficult to accommodate on spacecraft due to long antennas with their associated fields of view. Complicated antenna deployments are typically required.

Pencil-beam scatterometer systems were first described by Moore and others [13], with several variations proposed by Long [7], [9]. In contrast to fan-beam scatterometers, pencilbeam systems utilize a parabolic dish antenna which is mechanically spun to scan the ocean surface. This compact design is more readily accommodated on spacecraft without deployments. Because the antenna beam is conically scanned at a constant incidence angle, a wider contiguous swath is possible. This greatly increases ocean coverage relative to fanbeam systems. The constant relatively high incidence angles of the pencil-beam measurements also contribute to greater wind accuracy [3]. An associated advantage is that the model function relating $\sigma^{0}$ to wind velocity need be known only near the discrete incidence angles at which the measurements are made, rather than the broad range of incidence angles required by fan-beam systems. For these reasons, a pencilbeam architecture was chosen for SeaWinds.

In addition to the antenna concept, another important facet of the scatterometer design is the signal processing approach used to measure the backscattered signal and to estimate $\sigma^{0}$. Because the determination of ocean surface wind is a very sensitive function of the $\sigma^{0}$ measurement accuracy, a key consideration in the processor design is the minimization of the $\sigma^{0}$ error variance. Errors in the estimate of $\sigma^{0}$ are due to the random effects of radar signal fading and thermal noise, as well as calibration uncertainty. In general, $\sigma^{0}$ accuracy is improved by increasing the effective number of independent samples and the signal-to-noise ratio (SNR) associated with the measurement [2], [8]. Because $\sigma^{0}$ is a function of wind speed-backscatter cross section is low for low winds and high 


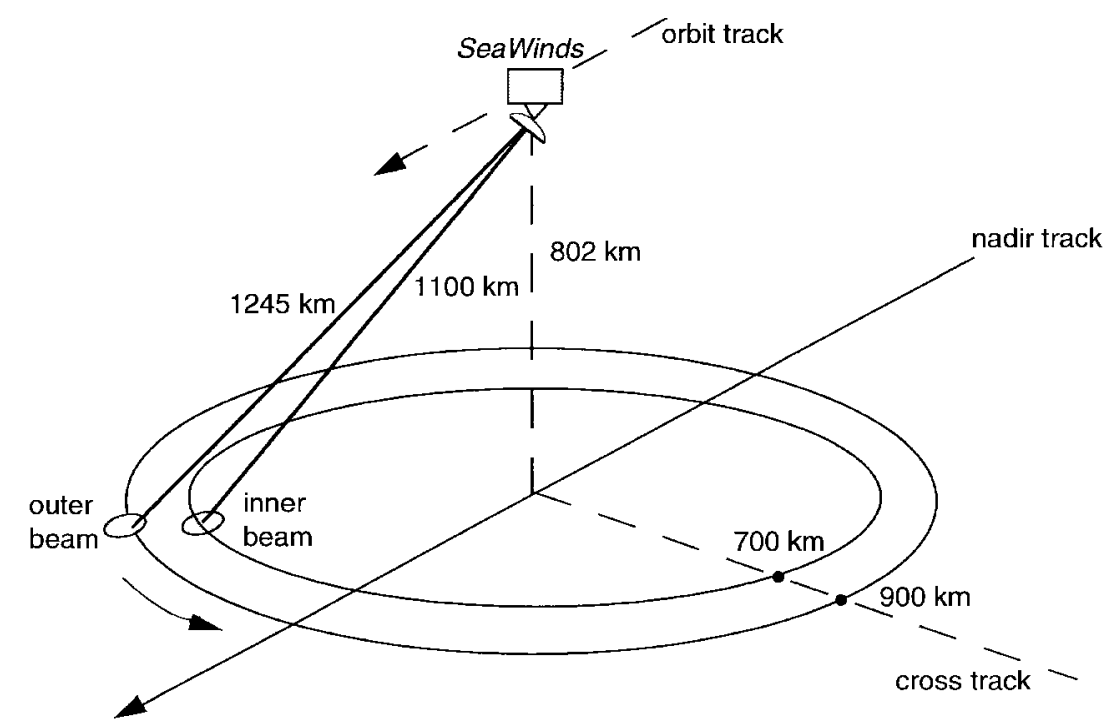

Fig. 1. SeaWinds measurement geometry.

for high winds - the SNR can vary considerably as different wind conditions are observed.

The issues and trade-offs encountered in selecting an optimal signal processing approach are generally different for fan-beam systems and pencil-beam systems. With fan-beam systems, $\sigma^{0}$ cell resolution is determined by either Doppler or range discrimination techniques, increasing overall processor complexity. Another challenge for fan-beam systems is that the antenna beam is very broad in one dimension, and the transmit energy is spread over a wide region. This may result in low SNR for the backscattered signal, particularly at low wind speeds. On the other hand, an advantage enjoyed by fan-beam systems is that, because the broad beam illuminates the entire swath simultaneously, relatively long integration times, and thus more independent samples, are possible.

Pencil-beam systems, by contrast, can use relatively simple processors because $\sigma^{0}$ resolution is completely defined by the antenna beam dimensions. Because energy is focused on a small region, SNR is generally higher for pencil-beam systems. Pencil-beam systems, however, have considerably less integration time available as the antenna footprint is quickly scanned from one location to another. A key challenge for the SeaWinds signal processing design is how to optimize performance by improving the effective number of independent samples. It is demonstrated that appropriate use of transmit pulse modulation can accomplish this goal.

The main body of this paper is divided into two distinct parts. In Section II the overall SeaWinds system design and $\sigma^{0}$ measurement approach are described. Included are brief descriptions of the instrument parameters, antenna scan approach, timing, and backscattered energy detection. Section III provides a more detailed look at the unique aspects of the SeaWinds signal processing design, and discusses the tradeoffs performed in order to achieve optimum $\sigma^{0}$ measurement performance. In particular, it is shown how modulation of the transmit pulse is employed to minimize $\sigma^{0}$ estimation error. A companion paper [11], which provides a theoretical basis for this technique, is referred to extensively.

\section{SEAWINDS SyStem DESIGN}

In preparation for a 1999 launch, the SeaWinds system is in an advanced stage of development. The SeaWinds instrument is being designed to meet the science requirements for wind retrieval [3], and to be consistent with the ADEOS-II orbit and accommodation constraints. In this section the functional aspects of the SeaWinds instrument design are presented. Included are brief descriptions of the selected radar parameters, antenna scan approach, timing, echo detection, and $\sigma^{0}$ calculation. In addition to providing a reference for the overall SeaWinds measurement technique, this description provides important background material against which the detailed signal processing tradeoffs in Section III are discussed. Additional information on the planned hardware implementation and ground data system can be found in Wu et al. [18].

\section{A. Antenna Characteristics and Measurement Geometry}

In order to determine the near surface wind, a scatterometer must obtain measurements of $\sigma^{0}$ at multiple azimuth angles for the same point on the ocean surface [16]. As illustrated in Figs. 1 and 2, SeaWinds accomplishes this using two conically scanned pencil beams. An approximately 1 $\mathrm{m}$ diameter parabolic dish antenna with two offset feeds is used to create both the "inner" and the "outer" beams. The inner beam maintains an "off-nadir" angle of $40^{\circ}$, and intercepts the ocean at a constant $46^{\circ}$ incidence angle. The outer beam has an off-nadir angle of $46^{\circ}$ with an incidence angle of $54^{\circ}$. The antenna is mechanically spun about the nadir axis to generate a conical scan. The scan azimuth angle is measured counter-clockwise with $0^{\circ}$ defined as the antenna pointing in the direction of the spacecraft motion. As the spacecraft moves in its orbit, the beams trace overlapping helical patterns on the Earth's surface. Each point within the inner $700 \mathrm{~km}$ of the swath is viewed from four different azimuth angles - twice by the outer beam looking forward then aft, and twice by the inner beam in the same fashion. In the outside edge of the swath, between cross track distances of 700 and $900 \mathrm{~km}$, each point on the ocean is viewed twice 
TABLE I

SeaWinds Antenna and Measurement Geometry Parameters

\begin{tabular}{|l|c|c|}
\hline Parameter & Inner Beam & Outer Beam \\
\hline Polarization & $\mathrm{H}$ & $\mathrm{V}$ \\
Elevation Angle & $40^{\circ}$ & $46^{\circ}$ \\
Surface Incidence Angle & $47^{\circ}$ & $55^{\circ}$ \\
Slant Range & $1100 \mathrm{~km}$ & $1245 \mathrm{~km}$ \\
$3 \mathrm{~dB}$ Beam Dimensions $(\mathrm{az} \times \mathrm{el})$ & $1.8^{\circ} \times 1.6^{\circ}$ & $1.7^{\circ} \times 1.4^{\circ}$ \\
$3 \mathrm{~dB}$ Footprint Dimensions $(\mathrm{az} \times \mathrm{el})$ & $34 \times 44 \mathrm{~km}$ & $37 \times 52 \mathrm{~km}$. \\
Peak Gain & $38.5 \mathrm{dBi}$ & $39 \mathrm{dBi}$ \\
Rotation Rate & \multicolumn{2}{|c|}{$18 \mathrm{rpm}$} \\
Along Track Spacing & $22 \mathrm{~km}$ & $22 \mathrm{~km}$ \\
Along Scan Spacing & $15 \mathrm{~km}$ & $19 \mathrm{~km}$ \\
\hline
\end{tabular}

by the outer beam only. The availability of four, rather than two, measurements over most of the total swath will enhance the ability of SeaWinds to unambiguously determine wind direction. Wind direction performance is further improved by using different polarizations for each beam [14]. The inner beam is horizontally polarized with respect to the ocean surface (the transmitted E-vector is parallel to the surface). The outer beam is vertically polarized.

Note that, unlike fan-beam systems, the azimuth angle "mix" of the $\sigma^{0}$ measurements going into the wind retrieval is not constant, but varies from nadir out to the edge of the swath. Near nadir the forward and aft measurements are approximately 180 degrees apart, while at the extreme edge of the swath the azimuth angle between the measurements approaches $0^{\circ}$. Thus, the wind retrieval performance of SeaWinds is observed to vary as a function of the distance from the nadir track, in general being optimum when the azimuth differences of the measurements are near $90^{\circ}$ [18]. But because the $\sigma^{0}$ measurements are obtained at a favorable high incidence angle over a continuous $1800 \mathrm{~km}$ swath, there is no distinct "nadir gap" where wind can not be retrieved. Such a wide swath will cover $90 \%$ of the ocean surface within 24 $\mathrm{h}$, an improvement over the NSCAT coverage of $77 \%$ in $24 \mathrm{~h}$.

The SeaWinds antenna rotation rate and measurement timing were chosen to obtain optimal sampling of the surface $\sigma^{0}$ and to meet host spacecraft dynamics requirements. The antenna rotation rate of $18 \mathrm{rpm}$ combined with the nominal transmitter pulse repetition frequency (PRF) of $92.5 \mathrm{~Hz}$ for each beam, produces a regular pattern of measurements on the surface. Fig. 3 shows this pattern for two consecutive rotations of the outer beam. The group of measurements displayed in Fig. 3 corresponds roughly to the rectangular region drawn in Fig. 2. Each transmit pulse event will obtain one measurement of $\sigma^{0}$, depicted in Fig. 3 as an ellipse whose dimensions are defined by the $3 \mathrm{~dB}$ contours of the antenna pattern projected on the surface. The "along scan" spacing of the measurements is a function of the scan rate and PRF, and is $15 \mathrm{~km}$ for the inner beam and $19 \mathrm{~km}$ for the outer beam. The "along track" displacement of the measurements is determined by the satellite ground speed of $6.6 \mathrm{~km} / \mathrm{s}$, and is $22 \mathrm{~km}$ for both beams. The footprint dimensions and spacing are consistent with the requirements to coregister the four azimuthal measurements, and to achieve near $50 \mathrm{~km}$ wind measurement resolution [3], [6]. Key parameters for each antenna beam and their associated footprints are summarized in Table I.

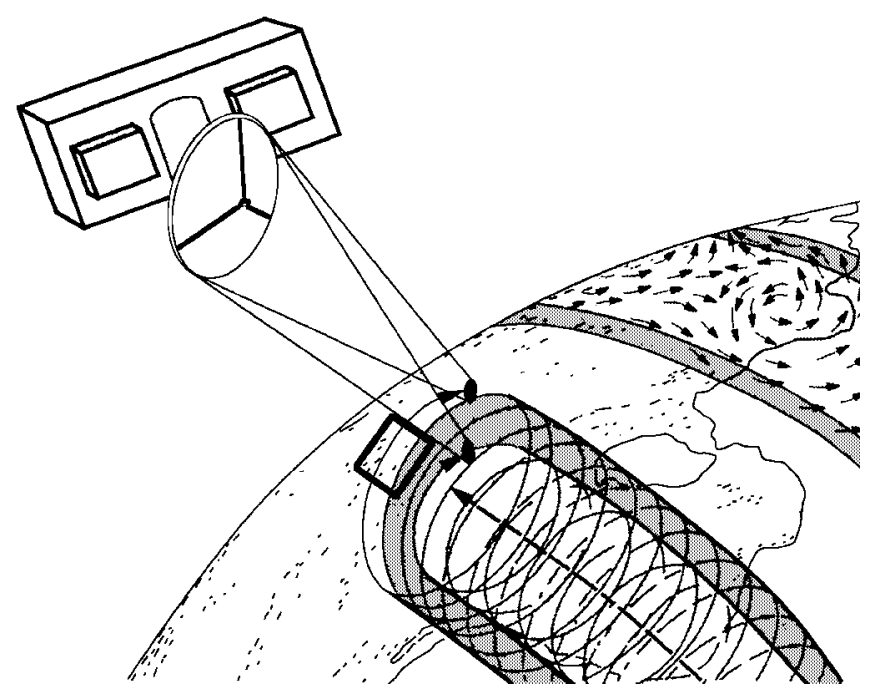

Fig. 2. SeaWinds scan pattern showing helixes traced by inner beam (light shade) and outer beam (dark shade) as spacecraft orbits. Expansion of region within box shown in Fig. 3.

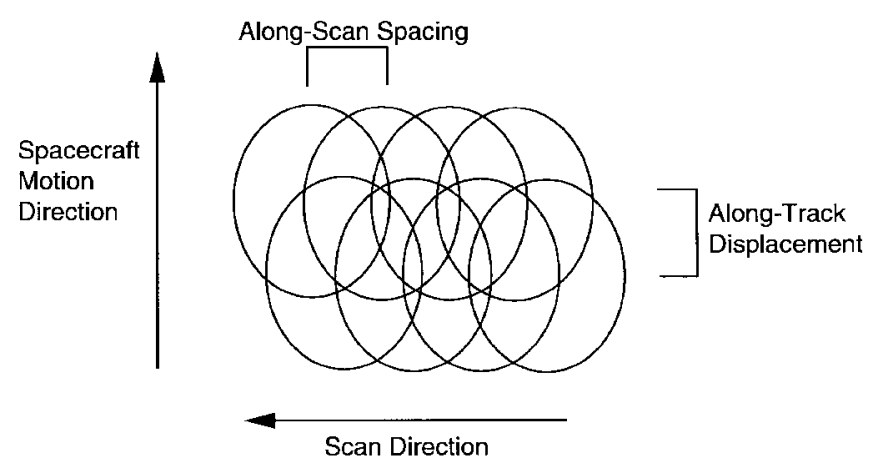

Fig. 3. Expansion of boxed region in Fig. 2 illustrating spacing of $\sigma^{0}$ measurements. Measurement footprints are defined by $3 \mathrm{~dB}$ contour of the antenna pattern.

\section{B. Radar Electronics Functional Design}

Fig. 4 depicts the basic design of the SeaWinds radar electronics and shows the transmit, receive, and detector functions. Upon command from the timing controller, the transmitter, which consists of a modulated signal generator driving a traveling wavetube (TWT) amplifier, issues a 1.5 ms duration, 110 Watt Ku-Band pulse. For reasons explained in Section III, the transmit pulse is MSK modulated to a bandwidth of approximately $40 \mathrm{kHz}$. The pulse is routed to either the inner or the outer beam and through a coaxial rotary joint to the spinning section of the antenna assembly. The echo return is likewise directed to the receiver where it is amplified, downconverted, and detected. A summary list of the key radar parameters is shown in Table II.

Due to the motion of the satellite relative to the Earth, a Doppler shift of between $\pm 500 \mathrm{kHz}$ is imparted to the echo return signal, depending on the antenna scan position. When the antenna is pointing forward or behind relative to the spacecraft motion (see Fig. 1), the Doppler shift is at a maximum or minimum. When the antenna is scanned perpendicular to the spacecraft ground-track, the shift is near zero. In the SeaWinds design, the Doppler shift is pre-compensated 


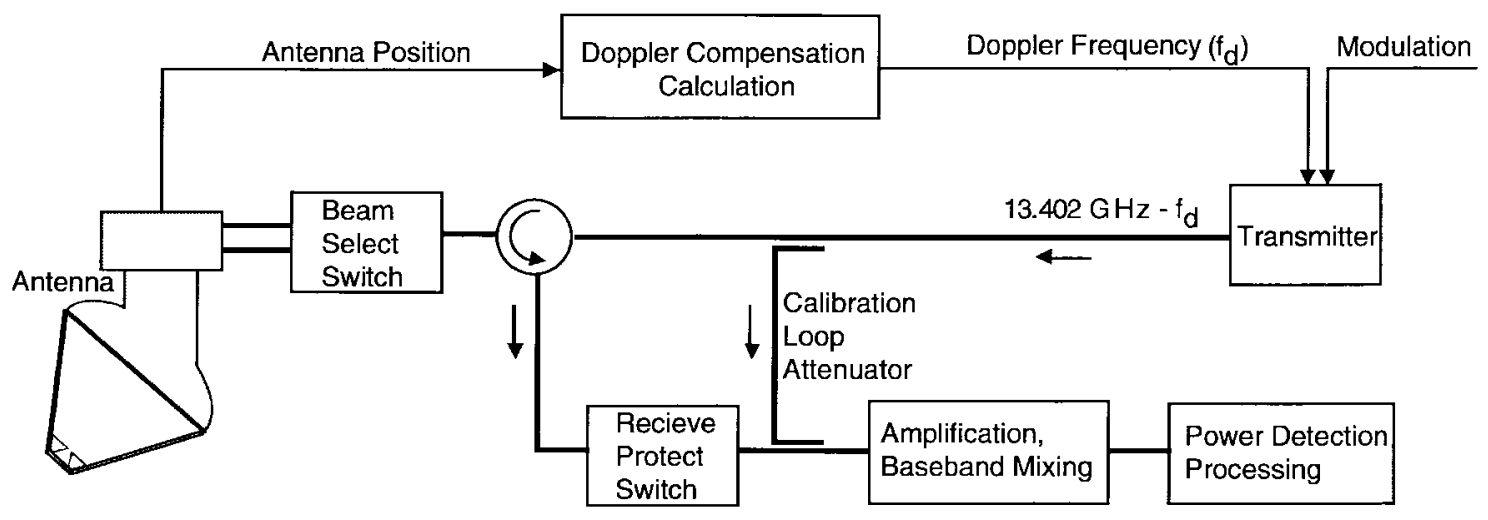

Fig. 4. Diagram of SeaWinds electronics functions.

Signal+Noise Measurement

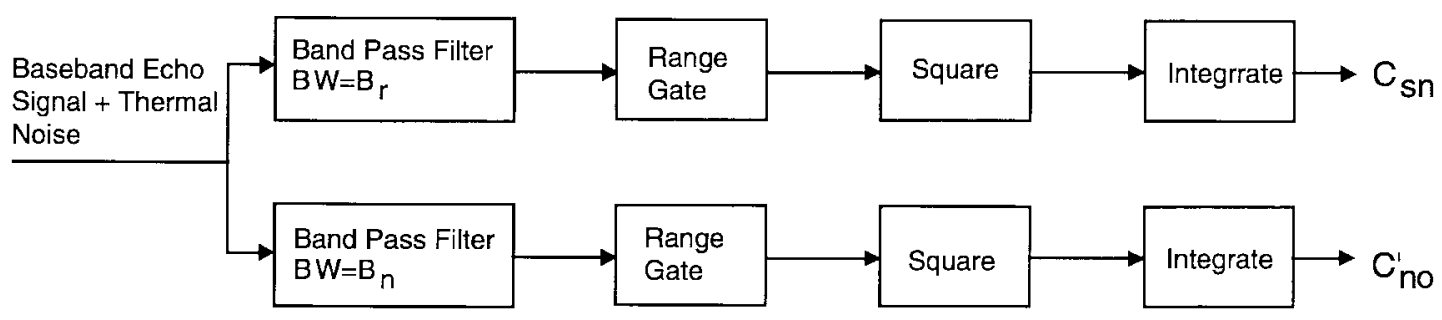

Noise-only Measurement

Fig. 5. Diagram of SeaWinds filtering and detector functions.

by tuning the transmit carrier frequency to $13.402 \mathrm{GHz}-f_{d}$, where $f_{d}$ is the expected frequency shift to be imparted to the return signal. The compensation frequency is computed by the SeaWinds on-board processor using the measured antenna position, orbit location, spacecraft velocity, and Earth rotation. Pre-compensating the transmit pulse for Doppler shift produces an echo signal that always occurs at the same center frequency, simplifying the RF down conversion and detector electronics. Note that the Doppler frequency could have been equivalently compensated by a variable IF frequency in the receiver, rather than a variable transmit frequency.

An important feature of any scatterometer system is the accurate calibration of the transmit power and receiver gain [15]. In the SeaWinds instrument design, these parameters are measured simultaneously by periodically injecting the transmit pulse, attenuated by a known amount, into the receiver. To avoid corruption by spurious leakage power during a "loopback" calibration event, a high loss receive protect switch is enabled.

\section{Echo Energy Detection and $\sigma^{0}$ Estimation}

The received signal at the detector consists of the return echo plus thermal noise. The thermal noise component is a combination of the instrument system noise and the Earth scene brightness temperature. In order to estimate $\sigma^{0}$, a measurement of the echo energy only is required. In scatterometer systems this is generally achieved by subtracting a separate measurement of the thermal noise floor-the "noiseonly" measurement - from the "signal+noise" measurement [2], [16].
TABLE II

SeaWinds Radar Electronics Parameters

\begin{tabular}{|l|c|}
\hline Parameter & Value \\
\hline Transmit Frequency & $13.402 \mathrm{GHz}$ \\
'Transmit Power & 110 Watts \\
'Iransinit PRF & $185 \mathrm{~Hz}(92.3 \mathrm{H} z$ each beam) \\
Transmit Pulse Length & $1.5 \mathrm{~ms}$ \\
Transmit Modulation & MSK, $T_{b}=15 \mu \mathrm{sec}$ \\
Receive Gate Length & $2.0 \mathrm{~ms}$ \\
Receive Gate Delay & $7.3 \mathrm{~ms}$ (inner beam) $8.3 \mathrm{~ms}$ (outer beam) \\
System Noisc Tempcrature & $740^{\circ} \mathrm{K}$ \\
\hline
\end{tabular}

A description of specific echo detection strategies for pencilbeam scatterometers is given in the companion paper [11]. For SeaWinds, a simple approach where the signal+noise and "noise-only" energies are measured simultaneously was selected. (Note that with the simultaneous detection approach, the "noise-only" measurement actually contains the echo energy as well. The term "noise-only," which is commonly used in scatterometry, is nonetheless retained here). As shown in Fig. 5, the signal+noise and noise-only energies, $C_{s n}$ and $C_{n o}^{\prime}$, are obtained by integrating the output of two separate filter operations. Fig. 6 shows a representation of the echo spectrum, thermal noise spectrum, and the magnitude response of the filters. The signal+noise filter has a bandwidth $B_{r}=80 \mathrm{kHz}$ and is centered on the echo return which has a $3 \mathrm{~dB}$ bandwidth of approximately $40 \mathrm{kHz}$. The noise-only filter has a much wider pass-band, $B_{n}=1 \mathrm{MHz}$, which overlaps the narrower signal+noise measurement band. The specific choice of filter bandwidths is based on measurement accuracy considerations, which is the subject of Section III. 


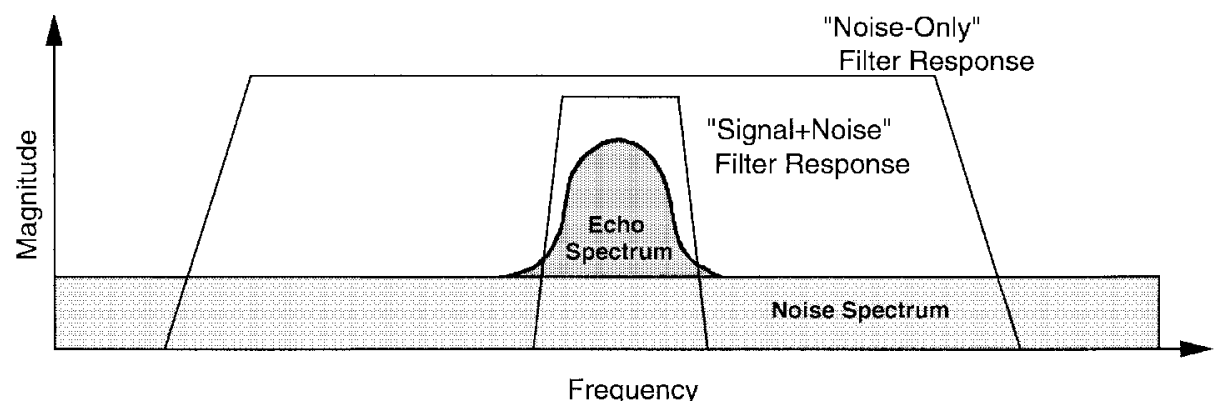

Fig. 6. Frequency domain representation of SeaWinds echo detection. Shown are the echo spectrum, white thermal noise spectrum, and the signal+noise and "noise-only" filter responses.

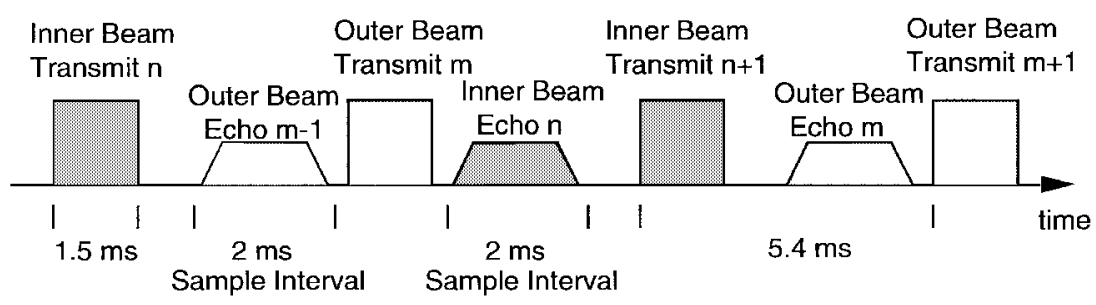

Fig. 7. SeaWinds transmit and detection timing.

Details of the transmit pulse and echo detection timing are shown in Fig. 7. The rectangular transmit pulses occur every $5.4 \mathrm{~ms}$ and alternate between the inner and outer beam. Note that this produces a PRF of approximately $92.5 \mathrm{~Hz}$ on each beam individually and $185 \mathrm{~Hz}$ overall. Due to the finite dimensions of the antenna footprint, the echo return "pulses" are not replicas of the transmit pulse but are dispersed in time and frequency. The dispersion in time spreads the echo by approximately $0.5 \mathrm{~ms}$, and is depicted in Fig. 7 by showing the echoes as trapezoids. The round trip flight times for the inner and outer pulses are 7.3 and $8.3 \mathrm{~ms}$, respectively, and thus each echo returns after the succeeding transmit pulse. The range gate interval during which both the signal+noise and noise-only measurements are accumulated is centered on the expected time of the echo return. In order to measure the entire energy of the echo, the range gate has a length $T_{r}=T_{n}=2$ ms.

The values $C_{s n}$ and $C_{n o}^{\prime}$ for each return pulse are telemetered to the ground where the echo energy and, ultimately, $\sigma^{0}$ are estimated. For the SeaWinds detection scheme, an expression for the echo energy estimate is obtained in a straightforward manner. Assuming a processor gain of unity, the expected values of $C_{s n}$ and $C_{n o}^{\prime}$ are written as

$$
\begin{aligned}
& \mathcal{E}\left[C_{s n}\right]=\rho_{E} E_{s}+B_{r} T_{r} n_{0} \\
& \mathcal{E}\left[C_{n o}^{\prime}\right]=E_{s}+B_{n} T_{r} n_{0}
\end{aligned}
$$

where $E_{s}$ is the expected energy in the echo return, $n_{0}$ is the one-sided thermal noise power spectral density, and $\rho_{E}$ is the fraction of the echo energy passed by the signal+noise filter. In the ideal case where the entire echo spectrum is captured by the filter, $\rho_{E}=1$. This is true of the noise-only filter. For the signal+noise filter, however, a small portion of the total energy from the sidelobes of the echo spectrum will be lost. The parameter $\rho_{E}$ is easily calculated by integrating the echo spectrum and filter response. [See (15) and associated discussion.].
Solving (1) and (2) for $E_{s}$, we obtain the following expression for the estimated echo energy $\widehat{E_{s}}$, given $C_{s n}$ and $C_{n o}^{\prime}$ :

$$
\widehat{E_{s}}=C_{s n} \frac{B_{n}}{\rho_{E}\left(B_{n}-B_{r}\right)}-C_{n o}^{\prime} \frac{B_{r}}{\rho_{E}\left(B_{n}-B_{r}\right)} .
$$

The well known scatterometer radar equation [16] is then applied to estimate $\sigma^{0}$

$$
\widehat{\sigma_{0}}=\frac{\widehat{E_{s}}}{X}
$$

where

$$
X=\frac{E_{t} \lambda^{2} G_{0}^{2} A_{c}}{(4 \pi)^{2} \bar{R}^{4}} .
$$

Here, $E_{t}$ is the total transmit pulse energy, $\lambda$ is the transmit carrier frequency, $G_{0}$ is the antenna pattern peak gain, $A_{c}$ is the effective illuminated footprint area, and $\bar{R}$ is the nominal slant range. For this paper, which specifically addresses the SeaWinds case, $A_{C}$ is defined as

$$
A_{c}=\frac{\bar{R}^{4}}{G_{0}^{2}} \int_{r, \omega_{d}} \frac{G_{t}\left(r, \omega_{d}\right) G_{r}\left(r, \omega_{d}\right)}{r^{4}} A_{r}\left(r, \omega_{d}\right) d r d \omega_{d}
$$

where $G_{t}$ is the antenna pattern at the time of transmit, $G_{r}$ is the antenna pattern at the time of echo return, $r$ is the range to each surface element, $A_{r}$ is the area of each surface element, and the integration is performed over range and Doppler on the surface. The transmit and receive antenna positions are specified separately in (6) because the antenna rotates approximately 0.8 degrees - a significant fraction of the beamwidth - during the pulse round trip flight. A precise formulation of the radar equation must take this offset into account [12]. 


\section{TRANSMIT MODULATION AND \\ PROCESSOR PARAMETER TRADEOFFS}

In this section we turn from a general overview of the SeaWinds instrument system to a more detailed discussion of a key design issue: the minimization of $\sigma_{0}$ measurement error. Here, general expressions for measurement variance derived in [11] are applied to the specific SeaWinds case in order to select the optimum transmit modulation and processor filter parameters.

An examination of (4) reveals that the error in determining $\sigma^{0}$ has two main components: the error associated with the estimate of $E_{s}$, and the error in the knowledge of the value of $X$. The process of measuring all the radar parameters which contribute to $X$ is referred to as scatterometer calibration. Calibration errors are largely systematic in that they are either biases or can be reduced by careful characterization of the on-orbit variations in the instrument radiometric parameters. Techniques for scatterometer calibration are beyond the scope of this paper but are discussed in detail elsewhere [10], [15]. The error associated with the estimate of $E_{s}$, however, is random and places a fundamental constraint on the theoretical wind performance of the scatterometer. It is this error that we seek to minimize here.

A metric, widely used in scatterometry, for evaluating the $E_{s}$ estimation error is the " $K_{p}$ " parameter

$$
K_{p}\left[\widehat{E_{s}}\right]=\left(\frac{\sqrt{\operatorname{var}\left[\widehat{E_{s}}\right]}}{E_{s}}\right)
$$

$K_{p}$ is the normalized standard deviation of $\widehat{E_{s}}$ due to radar fading and thermal noise effects. A general objective of scatterometer design is the minimization of $K_{p}$ [14]. In performing design trade-offs, it is useful to have a "rule of thumb" maximum value of $K_{p}$ to be achieved. Unfortunately, $K_{p}$ is a function of the signal-to-noise ratio which depends on the wind. Further, relating $K_{p}$ directly to the wind measurement performance can be difficult due to the nonlinearity in the wind retrieval process. As a result, we adopt the goal for SeaWinds that $K_{p}$ should be less than the geophysical modeling error - the percentage variation in $\sigma^{0}$ for a given wind velocity [10]. Such a criterion will insure that wind performance is limited not by the precision of the instrument, but only by our ability to relate the measured $\sigma^{0}$ 's to wind speed and direction via the model function. The magnitude of the geophysical modeling error at $\mathrm{Ku}$-band is not well known, but a value of $17 \%$ has been commonly used in other scatterometer performance analyses. For SeaWinds a design goal is thus to have $K_{p}<0.17$.

In the companion paper [11], the $K_{p}$ expression for a pencilbeam scatterometer is derived, and has the generalized form

$$
K_{p}\left[\widehat{E_{s}}\right]=\left(A+S B+S^{2} C\right)^{1 / 2}
$$

where $A, B$, and $C$ are rather complex functions of the transmit modulation, range and Doppler characteristics of the illuminated footprint, and the echo and noise filter bandwidths. $S$ is the noise-to-signal ratio (1/SNR) within the signal+noise filter band, and is defined as

$$
S=\frac{B_{r} T_{r} n_{0}}{E_{s}}
$$

where $E_{s}, B_{r}, T_{r}$, and $n_{0}$ are all as defined in the previous section. It is demonstrated in [11] that the " $A$ " term in (8), which is related to the effective number of independent samples, can be significantly decreased by appropriate modulation of the transmit pulse, thus decreasing $K_{p}$. A trade-off exists, however, because as the transmit pulse bandwidth increases, the bandwidth of the echo return necessarily increases and $B_{r}$ must subsequently widen to accommodate the larger echo bandwidth. A larger $B_{r}$ produces a larger $S$ via (9) which in turn tends to increase $K_{p}$. The objective here is to identify the modulation and filtering strategy which balances these effects and optimizes $K_{p}$ for SeaWinds. Our approach will be to first define the modulation scheme and the transmit pulse bandwidth, calculate the resulting echo return bandwidth and the $B_{r}$ required to accommodate it, and finally to evaluate (8) for the expected range of $S$ in order to select the optimal modulation bandwidth and filter parameters.

\section{A. Modulation Format and Transmit Pulse Spectrum}

As discussed in [11], for simple power detection modulation schemes which produce a "thumb-tack" shaped radar ambiguity function are good candidates for improving $K_{p}$. Due to its broad main lobe and low side lobe properties, as well as ease of implementation in hardware, a pseudorandom minimum phase shift keying (MSK) modulation format was selected for use with SeaWinds. Although all analysis in this paper is presented for the MSK case, similar techniques could be applied for other suitable modulation schemes.

When the transmit pulse length is much greater than the MSK modulation bit period, the one-sided energy spectral density, $S_{\mathrm{msk}}(\omega)$, of the transmitted signal can be shown to be [4]

$$
S_{\mathrm{msk}}(\omega)=16 \pi^{2} E_{t} T_{b}\left[\frac{\cos T_{b}\left(\omega-\omega_{c}+\omega_{d}\right)}{4 T_{b}^{2}\left(\omega-\omega_{c}+\omega_{d}\right)^{2}-\pi^{2}}\right]^{2}
$$

where $E_{t}$ is the total energy in the transmit pulse, $T_{b}$ is the modulation bit period, $\omega_{c}$ is the angular frequency of the transmit carrier $(2 \pi \times 13.402 \mathrm{GHz})$, and $\omega_{d}$ is the doppler precompensation applied to the transmit pulse $\left(2 \pi \times f_{d}\right)$. Note that the transmitted signal spectrum is centered at $\omega_{c}-\omega_{d}$. From (10) it can be shown that the $3 \mathrm{~dB}$ bandwidth of the transmit pulse, $B_{\mathrm{msk}}$, is approximately given by

$$
B_{\mathrm{msk}}=\frac{0.6}{T_{b}}
$$

\section{B. Echo Return Spectrum}

The spectrum of the echo return is different from that of the transmit pulse. The spectrum of the echo is a result of the interaction of the transmit signal with the ocean surface over the illuminated footprint. A well known technique for computing the echo return is to assume that the ocean surface is a collection of point scatterers [16]. The echo return is a summation of replicas of the transmit pulse scattered from each point on the surface, suitably weighted by the antenna 


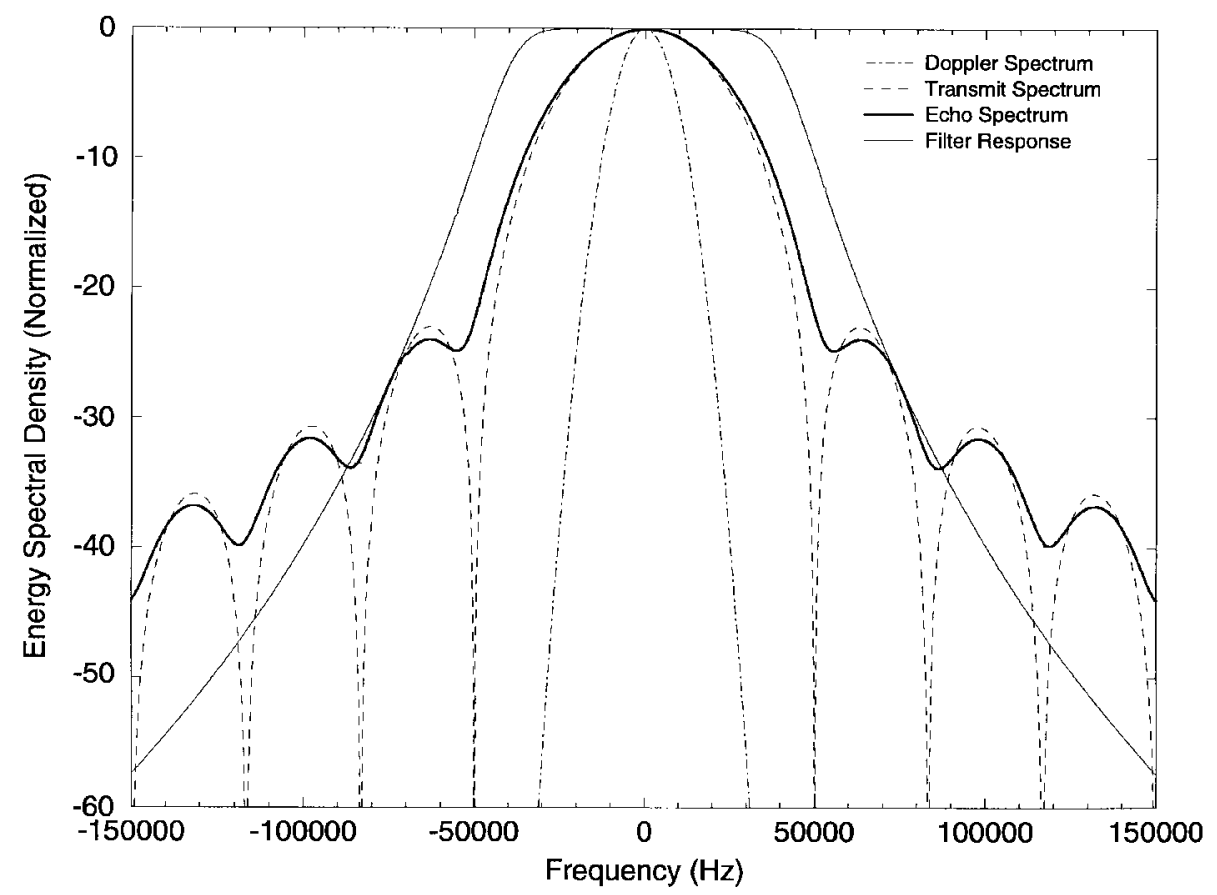

Fig. 8. Example transmit spectrum $\left(B_{\mathrm{msk}}=40 \mathrm{kHz}\right)$, Doppler spectrum (inner beam, scan azimuth $\left.90^{\circ}\right)$, and the resultant echo energy spectrum. Also shown is an example signal+noise filter magnitude response where $B_{r}=80 \mathrm{kHz}$.

pattern at each scattering element, and frequency shifted by the Doppler associated with each scatterer. The echo energy spectrum is likewise a summation of replicas of the transmit energy spectrum weighted by the antenna pattern and shifted by the Doppler frequency. Thus, the expected one-sided echo return spectrum at the receiver input, $E^{\prime}(\omega)$, can be written as a frequency domain convolution of the transmit spectrum and the footprint Doppler spectrum, $D(\omega)$

$$
E^{\prime}(\omega)=S_{\mathrm{msk}}(\omega) * D(\omega) .
$$

The Doppler spectrum represents the degree to which the transmit signal is spread in frequency due to the Doppler shift encountered over the illuminated area. Assuming a constant $\sigma^{0}$ over the illuminated footprint, the Doppler spectrum, $D(\omega)$, is defined as

$$
D(\omega)=\frac{\sigma^{0} \lambda^{2}}{(4 \pi)^{3}} \int \frac{G_{t}(r, \omega) G_{r}(r, \omega)}{r(r, \omega)^{4}} A_{r}(r, \omega) d r
$$

where the integration is performed over the iso-range lines within the footprint (see the treatment in [11]). Note that the Doppler spectrum is centered at $\omega_{d}$, and consequently $E^{\prime}(\omega)$ is centered at $\omega_{c}$. The downconverted, baseband echo spectrum incident on the detector, $E(\omega)$, is nominally centered at $0 \mathrm{~Hz}$ and is given by

$$
E(\omega)=E^{\prime}\left(\omega+\omega_{c}\right) .
$$

The shape and bandwidth of the Doppler spectrum, and thus the shape and bandwidth of the echo spectrum, depends on the selected antenna beam and azimuth angle. For SeaWinds, the $3 \mathrm{~dB}$ bandwidth of the Doppler spectrum, $B_{\mathrm{dop}}$, varies from about $9 \mathrm{kHz}$, for the outer beam scanned in the forward or aft direction ( 0 or $180^{\circ}$ azimuth), to $15 \mathrm{kHz}$, for the inner beam scanned to the side ( 90 or $270^{\circ}$ azimuth).
Fig. 8 is a graphical example of how the echo spectrum is calculated. Here, the transmit spectrum was computed from (10) assuming $B_{\mathrm{msk}}=40 \mathrm{kHz}$. The Doppler spectrum shown was produced by numerically evaluating (13) for the inner beam scanned to $90^{\circ}$ azimuth, and then removing the Dopppler center frequency. The resulting baseband echo spectrum was computed from (12). All spectra were normalized to a peak value of unity. Note that for the case shown, and indeed in all cases where $B_{\mathrm{msk}}>B_{\mathrm{dop}}$, the transmit spectrum is the dominant factor in determining the bandwidth of the echo return.

\section{Signal+Noise and Noise-Only Filter Bandwidths}

The signal+noise filter bandwidth, $B_{r}$, must be sufficiently wide to accommodate the echo return spectrum, yet as narrow as possible to minimize the amount of thermal noise passed. In the SeaWinds design process, two metrics are employed to assess the performance of the signal+noise filter: the fraction of the echo energy passed by the filter, and the Doppler compensation error. The fraction of the echo energy passed, $\rho_{E}$, is

$$
\rho_{E}=\frac{\int\left|H_{r}(\omega)\right|^{2} E(\omega) d \omega}{\int E(\omega) d \omega}
$$

where $\left|H_{r}(\omega)\right|^{2}$ is the magnitude response of the signal+noise filter, which is centered at $0 \mathrm{~Hz}$. The Doppler compensation error is caused by inexact pre-compensation of the transmit carrier for echo Doppler shift. Nominally, the Doppler shift imparted to the return signal is perfectly pre-compensated and the echo spectrum is centered in the signal+noise filter. In reality, antenna position uncertainty and spacecraft attitude 


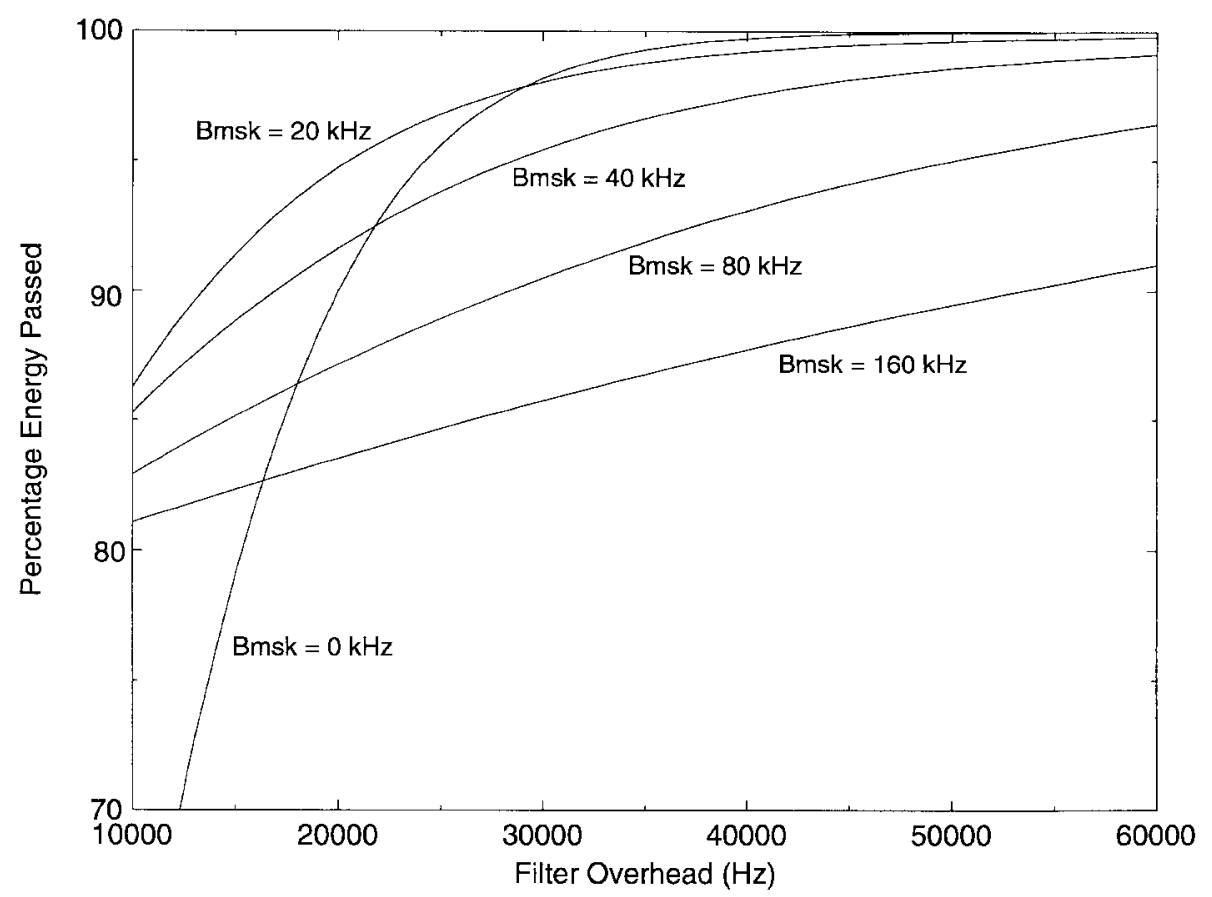

(a)

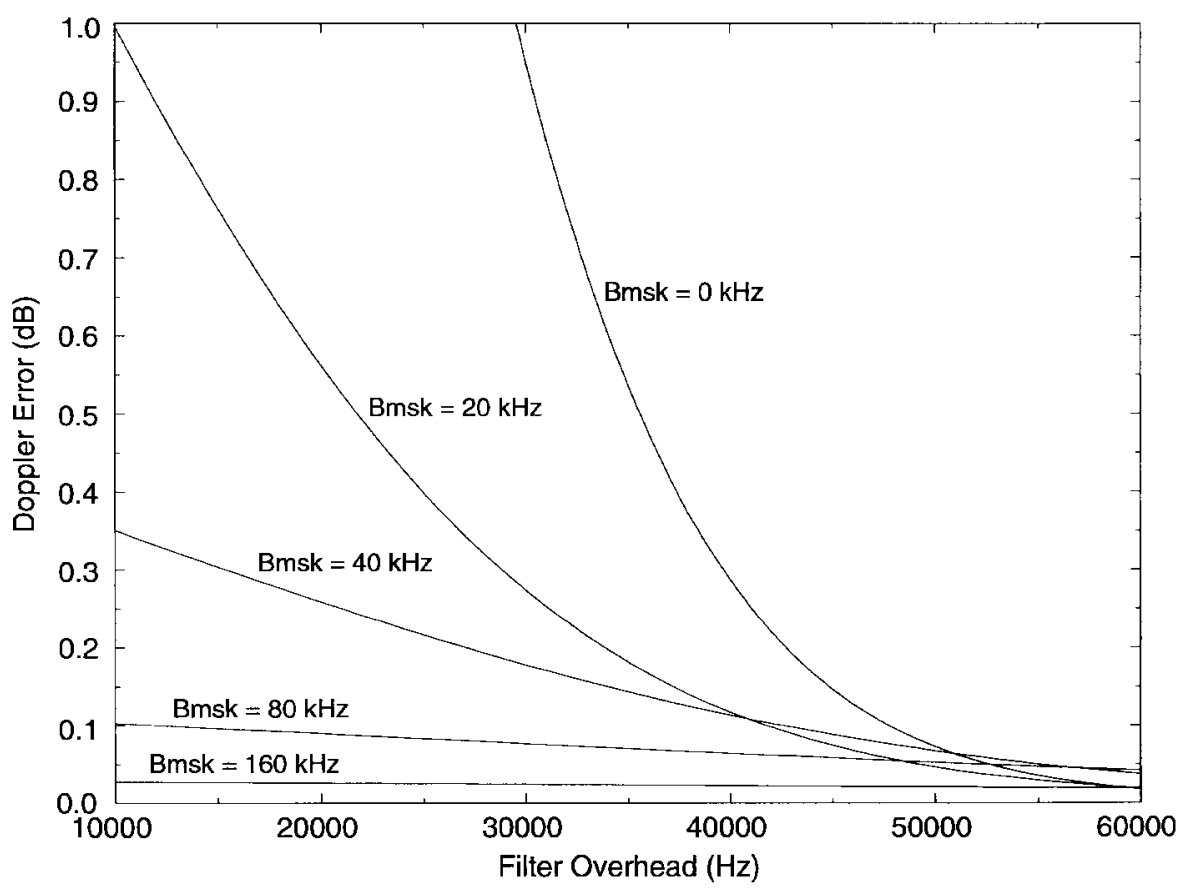

(b)

Fig. 9. (a) Percentage echo energy passed by signal+noise filter $\left(\rho_{E}\right)$ versus filter overhead $\left(B_{r}-B_{\mathrm{msk}}\right)$. (b) Doppler compensation error $\left(\Delta_{E}\right)$ versus filter overhead.

uncertainty lead to errors in Doppler tracking. The resultant error in detecting the echo energy, $\Delta_{E}$, is given by

$$
\Delta_{E}=\int\left|H_{r}(\omega)\right|^{2} E(\omega) d \omega-\int\left|H_{r}(\omega)\right|^{2} E\left(\omega-\omega_{\mathrm{err}}\right) d \omega
$$

where $\omega_{\text {err }}$ is the error associated with an inexact nulling of the Doppler center frequency, leading to an offset in the baseband echo spectrum. The filter bandwidth must be sufficiently wide to accommodate this "jitter" in the echo center frequency, without producing excessive error.

In examining the performance of the signal + noise filter, we shall define "filter overhead" as $B_{r}-B_{\mathrm{msk}}$, or the additional bandwidth of the filter over the $3 \mathrm{~dB}$ bandwidth of the transmit pulse. In Fig. 9(a) and (b), $\rho_{E}$ and $\Delta_{E}$ are calculated for five different values of $B_{\mathrm{msk}}$ as a function of filter overhead. In evaluating (15) and (16), $E(\omega)$ is computed with the $D(\omega)$ that results from the inner beam scanned to 90 degrees azimuth, maximizing the frequency dispersion due to Doppler. Note 


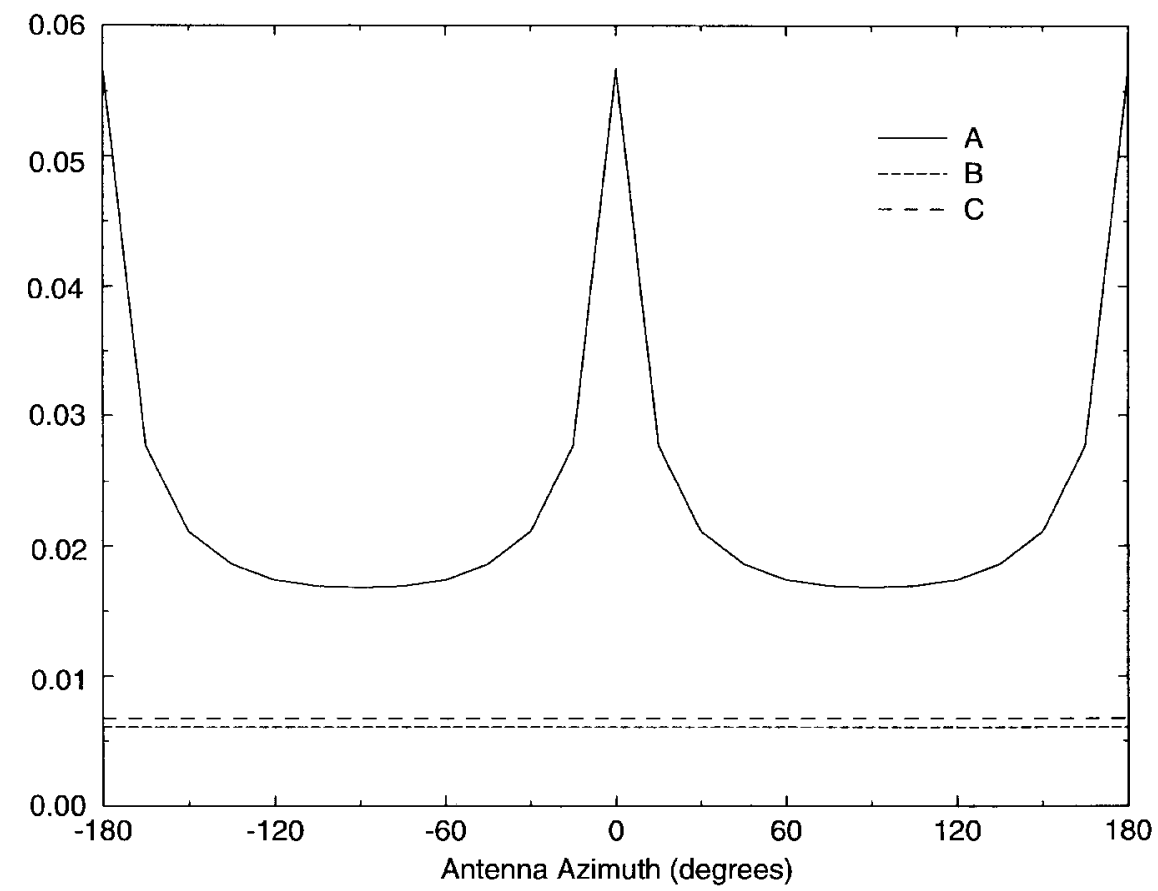

Fig. 10. Example of values of $K_{p}$ parameters $A, B$, and $C$ versus antenna azimuth angle for MSK modulation. For this case $B$ msk $=40$ kHz, $B_{r}=80 \mathrm{kHz}, B_{n}=1 \mathrm{MHz}$.

that in the case of $B_{\mathrm{msk}}=0 \mathrm{kHz}$, the transmit signal is the unmodulated carrier and the echo spectrum is the same as the Doppler spectrum. The filter magnitude response is a 5 th order Butterworth, an example of which for $B_{r}=80 \mathrm{kHz}$ is shown in Fig. 8. In computing $\Delta_{E}$, the Doppler compensation error was fixed at $10 \mathrm{kHz}\left(\omega_{\text {err }}=2 \pi \times 10^{4} \mathrm{rad} / \mathrm{s}\right)$, the anticipated maximum value for the SeaWinds design.

From Fig. 9(a) and (b) we can determine a relationship between the transmitted $B_{\mathrm{msk}}$ and the required $B_{r}$. To insure that a sufficient fraction of the echo energy is passed, we require that the filter be wide enough to pass $90 \%$ of the echo energy. To minimize frequency jitter induced errors in the measurement of echo return energy, we require $\Delta_{E}$ to be $<0.15 \mathrm{~dB}$. Applying these criteria to Fig. 9(a) and (b), we see that they are satisfied for a filter overhead of between 30 $\mathrm{kHz}$ and $50 \mathrm{kHz}$, depending on which $B_{\mathrm{msk}}$ is examined; the Doppler induced error being the primary factor for low values of $B_{\mathrm{msk}}$ and the percentage energy requirement being more important for large values of $B_{\mathrm{msk}}$. In our trade-off analyzes to find the optimum modulation bandwidth, we will find it useful to have a generalized relationship between $B_{\mathrm{msk}}$ and $B_{r}$. Such a relationship may be given by

$$
B_{r}=B_{\mathrm{msk}}+40 \mathrm{kHz} \text {. }
$$

Thus, we will size the bandwidth of the signal+noise filter to be $40 \mathrm{kHz}$ larger than the $3 \mathrm{~dB}$ bandwidth of the transmit pulse spectrum.

The role of the noise-only filter is to provide a separate measurement so that the thermal noise component can be subtracted from the signal+noise. As shown in the companion paper [11], the contribution to $K_{p}$ due to the noiseonly measurement is minimized for $B_{n} \gg B_{r}$. For the range of $B_{\mathrm{msk}}$ and $B_{r}$ considered in the SeaWinds trade-off analyzes $-B_{\mathrm{msk}}=0$ to $160 \mathrm{kHz}, B_{r}=40$ to $200 \mathrm{kHz}-\mathrm{a}$ noise-only filter bandwidth of $B_{n} \geq 1 \mathrm{MHz}$ will meet this condition. In general, however, the precise selection of the noise-only filter parameters is a less critical one than the selection of the signal+noise filter parameters.

\section{Selection of $B_{\mathrm{msk}}$ for $K_{p}$ Optimization}

Having specified the gate times $T_{r}$ and $T_{n}$, the modulation format, the required $B_{r}$ given $B_{\mathrm{msk}}$, and the required $B_{n}$; we can now evaluate (8) and address our primary goal of choosing the $B_{\text {msk }}$ which optimizes $K_{p}$. The parameters $A, B$, and $C$ are computed numerically as a function of antenna scan angle from the appropriate equations in the companion paper [11]. The correlation and cross-correlation integrals involved in the calculation of $A$ and $B$ are performed using the SeaWinds geometry and antenna patterns. The modulation function, $a(t)$, is generated using a maximal length, pseudo-random sequence which produces a $B_{\mathrm{msk}}$ consistent with (11). Because $K_{p}$ is calculated for a single pulse, $N_{p}=1$. As an example, Fig. 10 shows $A, B$, and $C$ as a function of azimuth for $B_{\mathrm{msk}}=40 \mathrm{kHz}, B_{r}=80 \mathrm{kHz}$, and $B_{n}=1 \mathrm{MHz}$. Note how, consistent with the observations in [11], the " $A$ " term varies significantly with azimuth for the selected modulation and detection scheme.

Noise-to-signal ratio, $S$, is an equally important consideration in the trade-off analysis. To insure that $K_{p}$ is optimized over the full range of possible ocean wind conditions, $S$ is calculated from (9) for three representative echo return energies corresponding to low wind $(3 \mathrm{~m} / \mathrm{s})$, moderate wind $(8 \mathrm{~m} / \mathrm{s})$, and high wind $(20 \mathrm{~m} / \mathrm{s})$ speed. These echo return strengths are shown in Table III, and were computed using the nominal SeaWinds instrument parameters and the average $\sigma^{0}$ as taken from the "SASS-2" model function [17]. The values of $E_{s}$ are given in dBJoules at the input to the SeaWinds receiver. 


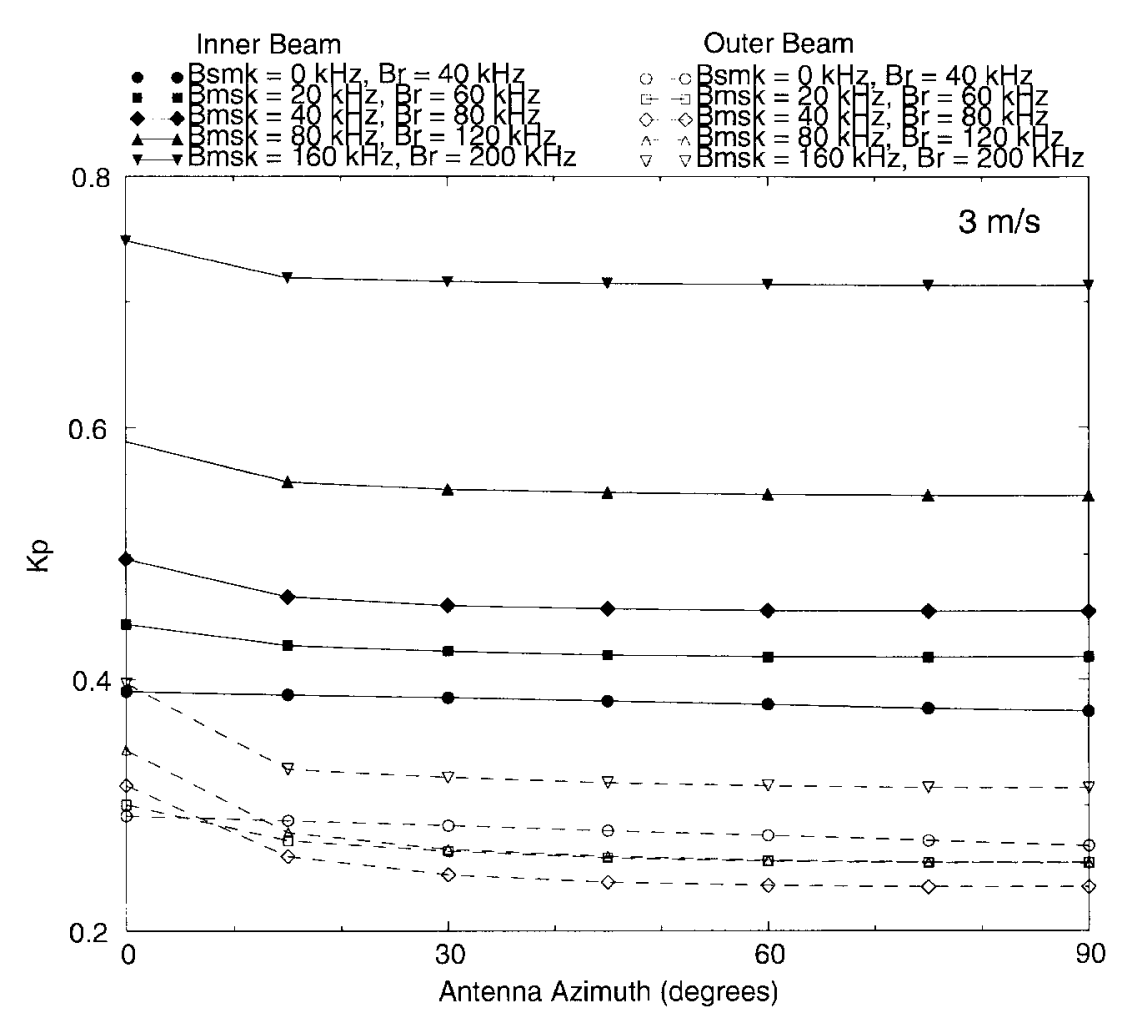

(a)

Fig. 11. $K_{p}$ versus azimuth for different values of $B_{\mathrm{msk}}$. (a) $3 \mathrm{~m} / \mathrm{s}$ wind. (b) $8 \mathrm{~m} / \mathrm{s}$ wind, (c) $20 \mathrm{~m} / \mathrm{s}$ wind.

TABLE III

SEAWinds Expected Echo ENergies

\begin{tabular}{|c||c|c||c|c|}
\cline { 2 - 5 } \multicolumn{1}{c|}{} & \multicolumn{2}{c||}{ Inner Beam } & \multicolumn{2}{c|}{ Outer Beam } \\
\hline Wind Speed & $\sigma^{0}(\mathrm{~dB})$ & $E_{s}$ (dBJoules) & $\sigma^{0}(\mathrm{~dB})$ & $E_{s}$ (dBJoules) \\
\hline $3 \mathrm{~m} / \mathrm{s}$ & -32 & -184 & -27 & -179 \\
$8 \mathrm{~m} / \mathrm{s}$ & -23 & -175 & -20 & -172 \\
$20 \mathrm{~m} / \mathrm{s}$ & -14 & -167 & -14 & -167 \\
\hline
\end{tabular}

The thermal noise power spectral density, $n_{0}$, is assumed to be $-200 \mathrm{dBW} / \mathrm{Hz}$.

In Fig. 11(a)-(c) $K_{p}$ versus azimuth angle for a range of $B_{\text {msk }}$ is plotted for each beam at the three representative wind speeds. Because the effects of modulation are nearly symmetric with azimuth quadrant, only the azimuth range between $0-90^{\circ}$ is shown. Note that selected transmit modulation significantly reduces $K_{p}$ for most of the swath, but offers little improvement for scan angles pointed forward or aft of the spacecraft-near 0 and $180^{\circ}$. The curves also exhibit an important trend as a function of wind speed. At the high wind speeds where $S$ is small, the " $A$ " term in (8) dominates and a larger $B_{\text {msk }}$ leads to lower $K_{p}$. Thus, in a high SNR environment, measurement accuracy can be improved by modulating the signal and consequently increasing the effective number of independent samples. At lower wind speeds, however, $K_{p}$ can actually increase for larger $B_{\mathrm{msk}}$. This is particularly evident for the inner beam case in Fig. 11(a). Here, the benefit derived from modulating the signal is overcome by the deleterious effect of increasing $B_{r}$ and passing more thermal noise to the detector.

An evaluation of the curves in Fig. 11 led to the selection of $B_{\mathrm{msk}}=40 \mathrm{kHz}$ and $B_{r}=80 \mathrm{kHz}$ for SeaWinds. Relative to the unmodulated case, $B_{\mathrm{msk}}=40 \mathrm{kHz}$ significantly improves $K_{p}$ performance over most of the swath and for most wind conditions. Lower values of $B_{\text {msk }}$ produced inferior performance at high and moderate wind speeds, in general failing the meet the performance goal of $K_{p}<0.17$. Higher values of $B_{\mathrm{msk}}$ were judged to produce undesirably large $K_{p}$ at low speeds. Due to the greater scientific importance of high wind measurements, a degree of performance degradation at low wind speeds, such as that experienced with $B_{\mathrm{msk}}=40 \mathrm{kHz}$ for the inner beam at $3 \mathrm{~m} / \mathrm{s}$, was deemed acceptable. Note that for the values of $S$ achievable with the SeaWinds design parameters, the goal of $K_{p}<0.17$ is not possible at the very lowest wind speeds for any modulation bandwidth. Despite being " $K_{p}$ limited" rather than "model function limited" in the less critical low wind speed regime, SeaWinds performance simulations indicate that the desired measurement accuracies will still be met [18].

\section{E. Simultaneous versus Nonsimultaneous Detection}

As discussed in [11], the determination of $E_{s}$ can be made with either of two different detection schemes, 1) signal+noise energy and noise-only energy are measured at the same time and in the same band (termed "simultaneous" detection), or 2) echo and noise energy are measured at different times or in different bands ("nonsimultaneous" detection). In the case of nonsimultaneous detection, the echo and noise measurements are independent; in the overlapping case they are not.

The minimum $K_{p}$ is achieved for nonsimultaneous detection. For SeaWinds, however, there are distinct advantages to using simultaneous detection. Because the antenna is scanning, the scene brightness temperature, which contributes 


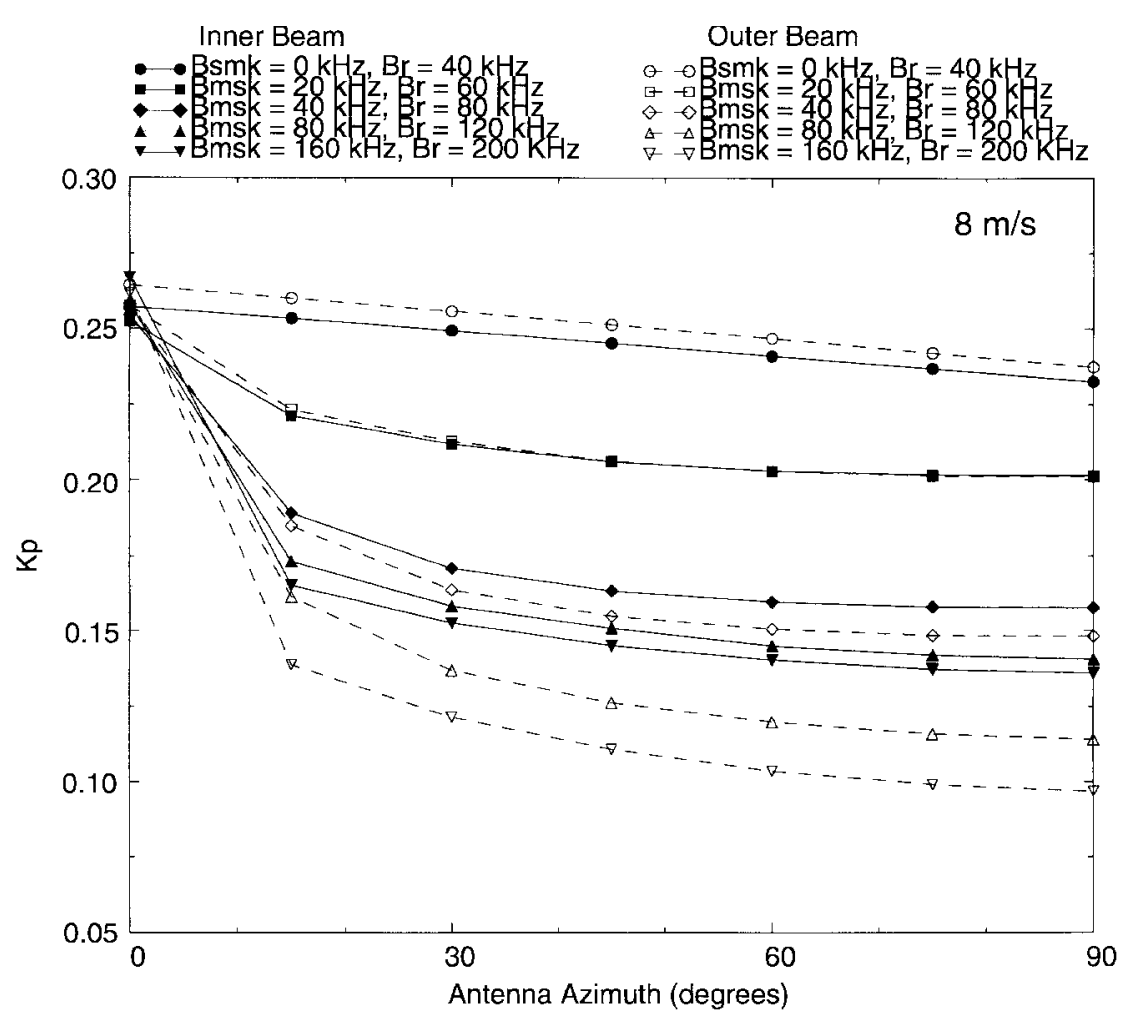

(b)

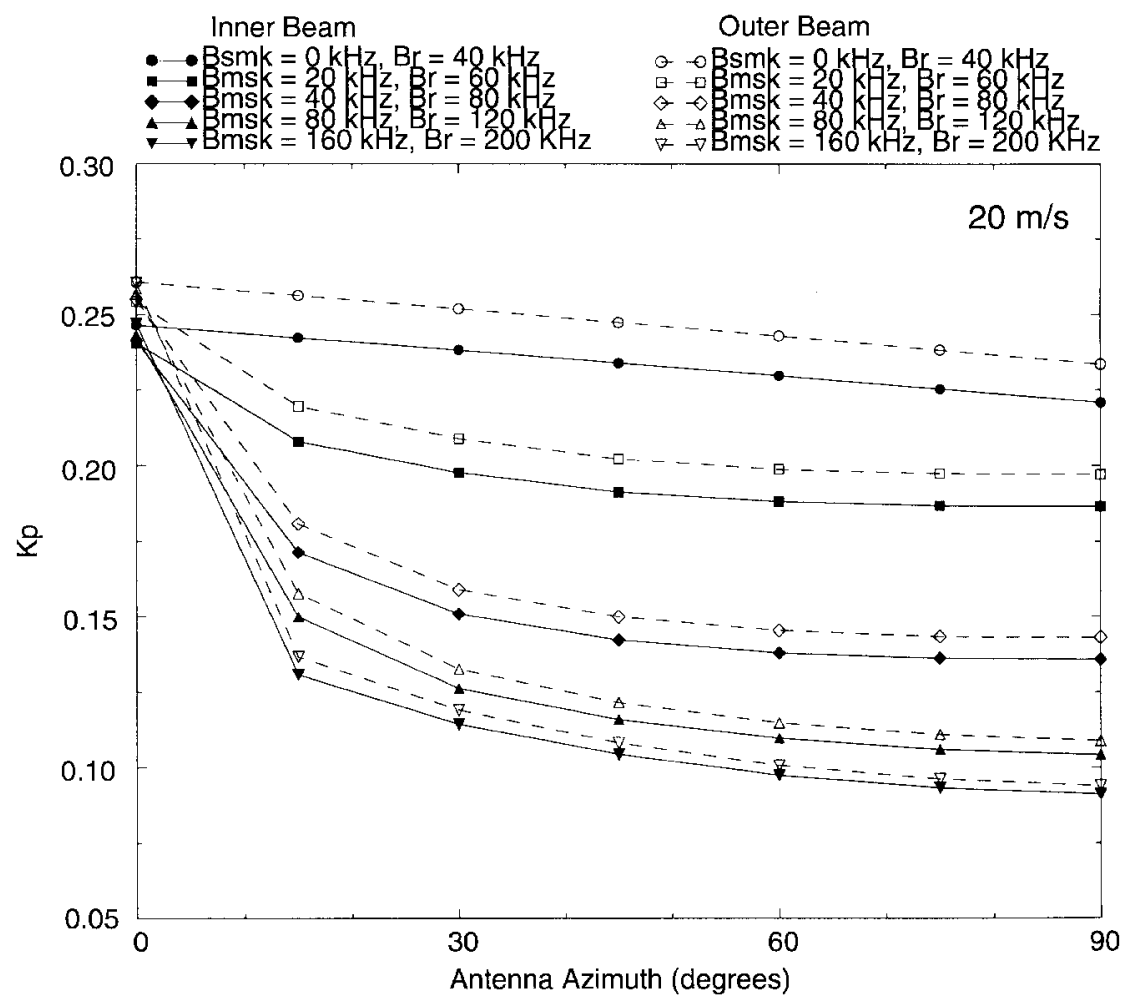

(c)

Fig. 11. (Continued.) $K_{p}$ versus azimuth for different values of $B_{\mathrm{msk}}$. (b) $8 \mathrm{~m} / \mathrm{s}$ wind, (c) $20 \mathrm{~m} / \mathrm{s}$ wind.

to the noise floor we are attempting to subtract out, may change quickly (see Fig. 3). This will be particularly true near ocean/land or ocean/ice boundaries. Thus, a simultaneous measurement of the noise energy will eliminate potential biases due to spatial mislocation with respect to the echo measurement. An additional reason to desire simultaneous detection is to simplify the hardware; the timing and IF frequencies are the same for the two measurements.

To assess the degree of degradation in $K_{p}$ suffered by using a simultaneous detection scheme, $K_{p}$ for the simultaneous 
and nonsimultaneous cases were compared for the selected SeaWinds modulation and filter parameters. It was found that the percentage $K_{p}$ increase due to using the simultaneous scheme was less than $3 \%$ - an acceptable amount. This is due to the fact that, as pointed out in [11], $K_{p}$ for the simultaneous case approaches that of the nonsimultaneous case when $B_{n} \gg B_{r}$. This is true for SeaWinds as $B_{n} \geq 1 \mathrm{MHz}$ and $B_{r}=80 \mathrm{kHz}$.

\section{SUMMARY}

The functional design and relative advantages of the SeaWinds scatterometer have been described. Because SeaWinds employs a compact dish antenna rather than multiple fanbeam antennas, the instrument is more easily accommodated on spacecraft than previously flown scatterometers. The SeaWinds swath is $1800 \mathrm{~km}$ wide and will allow global ocean surface winds to be measured more frequently.

It was demonstrated how SeaWinds $\sigma^{0}$ measurement variance is minimized by employing modulation of the transmit pulse. For the SeaWinds system there is a trade-off between performance at higher wind speeds, which is enhanced by modulation, and performance at lower wind speeds, which may be degraded by modulation. The selected modulation and filter parameters- $B_{\mathrm{msk}}=40 \mathrm{kHz}, B_{r}=80 \mathrm{kHz}, B_{n} \geq 1$ $\mathrm{MHz}$ - insure that measurements at moderate and high wind speeds are significantly improved, whereas only slight degradation occurs at low wind speeds. At high wind speeds, which are of intense scientific interest, $\sigma^{0}$ measurement accuracy is improved by $40 \%$ over the no-modulation case. This improved $\sigma^{0}$ measurement accuracy will bring about a corresponding improvement in wind retrieval accuracy.

Due to the inherent advantages of the pencil-beam approach, future scatterometer systems which follow SeaWinds are likely to use a similar architecture. Although we have concentrated on the specific SeaWinds design, it is hoped that the design issues and trade-offs discussed will be of general utility for other spaceborne scatterometers.

\section{REFERENCES}

[1] E. P. W. Attema, "The active microwave instrument on-board the ERS-1 satellite," in Proc. IEEE, vol. 79, pp. 791-799, June 1991.

[2] R. Fisher, "Standard deviation of scatterometer measurements from space," IEEE Trans. Geosci. Electron., vol. GE-10, Apr. 1972.

[3] M. H. Freilich, D. G. Long, and M. W. Spencer, "SeaWinds: A scanning scatterometer for ADEOS II-Science overview," in Proc. Int. Geosci. Remote Sensing Symp., Pasadena, CA, Aug. 8-12, 1994, pp. 960-963.

[4] S. Haykin, Communication Systems, 2nd ed. New York: Wiley 1983.

[5] W. L. Jones, L. C. Schroeder, D. H. Boggs, E. M. Bracalente, R. A. Brown, G. J. Dome, W. J. Pierson, and F. J. Wentz, "The SEASAT-A satellite scatterometer: The geophysical evaluation of remotely sensed wind vectors over the ocean," J. Geophys. Research, vol. 87, no. C5, Apr. 30, 1982.

[6] D. G. Long, "Wind measurement resolution for a scanning pencil beam scatterometer," in Proc. Int. Geosci. Remote Sensing Symp., Pasadena, CA, Aug. 8-12, 1994, pp. 948-952.

[7] _ _ASCAT: A lightweight, low-cost scatterometer," Microwave Instrumentation for Remote Sensing of the Earth, J. C. Shiue, Ed., Proc. SPIE 1935, pp. 28-38, Orlando, FL, Apr. 13-14, 1993.
[8] D. G. Long, C-Y Chi, and F. K. Li, "The design of an onboard digital doppler processor for a spaceborne scatterometer," IEEE Trans. Geosci. Remote Sensing, vol. 26, pp. 869-878, Nov. 1988.

[9] D. G. Long, M. H. Freilich, D. F. Leotta, and D. E. Noon, "A scanning scatterometer for the Eos polar platform," Proc. Int. Geosci. Remote Sensing Symp., Washington, D.C., May 20-24, 1990, pp. 2447-2450.

[10] D. G. Long and G. B. Skouson, "Calibration of spaceborne scatterometers using tropical rainforests," IEEE Trans. Geosci. Remote Sensing, vol. 34, pp. 413-424, Mar. 1996.

[11] D. G. Long and M. W. Spencer, "Radar backscatter measurement accuracy for a spaceborne pencil-beam wind scatterometer with transmit modulation," IEEE Trans. Geosci. Remote Sensing, this issue, pp. XXX.

[12] R. K. Moore, "Effect of pointing errors and range on performance of dual-pencil-beam scatterometers," IEEE Trans. Geosci. Remote Sensing, vol. GRS23, pp 901-905, Nov. 1985.

[13] R. K. Moore, R. G. Kennett, and F. K. Li, "Performance of a scanning pencil-beam spaceborne scatterometer for ocean wind measurements," in Proc. Int. Geosci. Remote Sensing Symp., Edinburgh, Scotland, Aug. 1988 , pp. $563-564$.

[14] F. Naderi, M. H. Freilich, and D. G. Long, "Spaceborne radar measurement of wind velocity over the ocean-An overview of the NSCAT scatterometer system," in Proc. IEEE, pp. 850-866, vol. 79, June 1991.

[15] M. W. Spencer, W. Tsai, S. Yueh, and G. Neumann, "NASA scatterometer calibration philosophy and approach," Microwave Instrumentation for Remote Sensing of the Earth, James C. Shiue, Ed., in Proc. SPIE 1935, pp. 63-73, Orlando, FL, Apr. 13-14, 1993.

[16] F. T. Ulaby, R. K. Moore, and A. K. Fung, Microwave Remote Sensing-Active and Passive. Reading, MA: Addison-Wesley, 1981.

[17] F. J. Wentz, S. Peteherych, and L. A. Thomas, "A model function for ocean radar cross sections at $14.6 \mathrm{GHz}$, , J. Geophysical Res., vol. 89, pp. 3689-3704, 1984.

[18] C. Wu, J. Graf, M. Freilich, D. G. Long, M. Spencer, W. Tsai, D. Lisman, and C. Winn, "The SeaWinds scatterometer instrument," in Proc. Int. Geosci. Remote Sensing Symp., Pasadena, CA, Aug. 8-12, 1994, pp. 1511-1515.

Michael W. Spencer, for a photograph and biography, see this issue, p. 114.

Chialin Wu was born in 1947. He received the B.S. degree in physics from the National Taiwan University in 1969, and the M.S. and Ph.D. degrees in electrical engineering from Purdue University in 1972, and 1975, respectively.

While at Purdue University he did research in imaging sonar and remote sensing. In the remote sensing area, he was involved in the early NASA ERTS satellite multispectral pattern recognition and image processing development with the Laboratory for Application of Remote Sensing. In 1974, he joined the Jet Propulsion Laboratory, engaging in digital signal processing research and system engineering for the Seasat synthetic aperture radar (SAR). $\mathrm{He}$ developed the digital signal processing algorithm and also the operational processing system for producing digital SAR imagery from data acquired from the 1978 Seasat mission. He worked on the Project of Magellan Mission to Venus, and served as the Deputy Radar Manager from 1989 to 1992 during the satellite cruise and mapping operation period. His responsibility on the Magellan Mission is radar system engineering and new development. He developed the interferometry scheme for the Magellan burst mode radar over adjacent orbits as well as over orbits separated by one Venus revolution of 243 days. Since 1992, he has served on a spaceborne radar scatterometer project, SeaWinds, as the Experiment Engineer, responsible for the instrument requirements and end-to-end performance.

Dr. Wu received the NASA Exceptional Service Medal for the SAR processor development in 1981. He was the co-investigator and co-inventer of the patent on digital SAR interferometry for topography and change detection.

David G. Long, for a photograph and biography, see this issue, p. 114. 